\title{
Non-Gaussianity from self-ordering scalar fields
}

\author{
Daniel G. Figueroa, ${ }^{1,2}$ Robert R. Caldwell, ${ }^{3}$ and Marc Kamionkowski ${ }^{4}$ \\ ${ }^{1}$ Department of Physics, CERN - Theory Division, CH-1211 Geneva 23, Switzerland \\ ${ }^{2}$ Instituto de Física Teórica UAM/CSIC, Universidad Autónoma de Madrid, 28049 Madrid, Spain \\ ${ }^{3}$ Department of Physics and Astronomy, Dartmouth College, Hanover, New Hampshire 03755, USA \\ ${ }^{4}$ California Institute of Technology, Mail Code 350-17, Pasadena, California 91125, USA
}

(Received 2 March 2010; published 4 June 2010)

\begin{abstract}
The Universe may harbor relics of the post-inflationary epoch in the form of a network of self-ordered scalar fields. Such fossils, while consistent with current cosmological data at trace levels, may leave too weak an imprint on the cosmic microwave background and the large-scale distribution of matter to allow for direct detection. The non-Gaussian statistics of the density perturbations induced by these fields, however, permit a direct means to probe for these relics. Here we calculate the bispectrum that arises in models of self-ordered scalar fields. We find a compact analytic expression for the bispectrum, evaluate it numerically, and provide a simple approximation that may be useful for data analysis. The bispectrum is largest for triangles that are aligned (have edges $k_{1} \simeq 2 k_{2} \simeq 2 k_{3}$ ) as opposed to the local-model bispectrum, which peaks for squeezed triangles $\left(k_{1} \simeq k_{2} \gg k_{3}\right)$, and the equilateral bispectrum, which peaks at $k_{1} \simeq k_{2} \simeq k_{3}$. We estimate that this non-Gaussianity should be detectable by the Planck satellite if the contribution from self-ordering scalar fields to primordial perturbations is near the current upper limit.
\end{abstract}

DOI: 10.1103/PhysRevD.81.123504

PACS numbers: 98.80.-k, 11.30.Fs, 98.80.Cq

\section{INTRODUCTION}

A wealth of precise cosmological data are in good agreement with the predictions of the simplest single-field slow-roll (SFSR) inflationary models [1]. Still, no theorist considers these as anything more than toy models. Realistic models must surely be more complicated, and they generically predict that there should arise, at some point, observable phenomena that depart from the predictions of SFSR inflation. Some possible directions for physics beyond the SFSR approximation include multifield models [2,3] and inflaton models with nonstandard kinetic terms [4]. There has also been investigation of the consequences of topological defects [5] produced toward the end of or after inflation [6].

If inflation was followed by a transition associated with the breaking of a global $O(N)$ symmetry, then self-ordering scalar fields (SOSFs) are another possibly observable early-Universe relic, even if there are no topological defects (i.e., if $N>4$ ). Here, the alignment of the scalar field as the Universe expands gives rise to a scale-invariant spectrum of isocurvature perturbations, without topological defects [7]. Sample variance on the current data limit these perturbations to contribute no more than $\sim 10 \%$ of large-angle cosmic-microwave-background (CMB) anisotropy power $[8,9]$. SOSF models are parametrized simply by the number $N$ of scalar fields and the vacuum expectation value $v$. The CMB constraint implies $\left(v / N^{1 / 4}\right) \lesssim 5 \times$ $10^{15} \mathrm{GeV}$, as we explain below. At this low amplitude, it is unlikely that any surviving relics leave a distinct imprint on the CMB power spectrum [10].

In recent years, non-Gaussianity has been developed as a novel tool to investigate beyond-SFSR physics $[11,12]$.
SFSR models do not predict that primordial perturbations should be Gaussian, but the departures from Gaussianity that they predict are unobservably small [13-15]. Multifield models [2], such as curvaton models [3], string-inspired Dirac-Born-Infeld (DBI) $[4,16]$ models, and models with features in the inflaton potential $[14,17]$ can all produce larger, and possibly observable, deviations from non-Gaussianity. For example, the detailed shape (triangle dependence) of the bispectrum may also help distinguish these different scenarios. The "local-model" bispectrum, like that which arises in curvaton and multifield models, has a very different shape dependence than "equilateral-model" bispectra, like those in DBI models. Non-Gaussianity can be sought in the CMB [18], largescale structure (LSS) [19], and the abundances and properties of gravitationally bound objects [20] or voids [21]. Biasing may significantly amplify the effects of nonGaussianity [22] in the galaxy distribution.

The energy-density perturbations in self-ordering scalar fields are quadratic in the scalar-field perturbation, which may itself be approximated as a Gaussian field. The density perturbations induced by SOSFs are thus expected to be highly non-Gaussian [7,23,24], even in the absence of topological defects. It is thus plausible that the nonGaussianity induced by SOSFs might be detectable, even if they provide only a secondary contribution to primordial perturbations.

In this paper, we perform the first calculation of the full shape (triangle) dependence of the bispectrum from SOSFs. We follow the formalism for non-Gaussianity developed in Ref. [23]. We find considerably simplified formulas for the bispectrum, evaluate them numerically, and find a simple approximation to aid in data-analysis efforts. 
We estimate the current non-Gaussianity constraint to the model parameter space and find it to be comparable to that from the upper limit to isocurvature perturbations from CMB fluctuations.

The plan of this paper is as follows: In Sec. II, we define the model, write the scalar-field equations of motion, show that the dynamics are those of a nonlinear-sigma model, and introduce the large- $N$ scaling limit for the nonlinearsigma model. In Sec. III, we write the relation between the matter-density perturbation and the scalar-field perturbation. In Sec. IV, we derive the power spectrum for density and curvature perturbations, discuss the normalization, and derive current constraints to the $v-N$ parameter space from upper limits to the SOSF contribution to CMB fluctuations. In Sec. V, we discuss the calculation of the bispectrum, the central focus of this paper. We present a simplified version, our Eq. (26), of the matter-bispectrum expression in Ref. [23], evaluate it numerically, and provide a simple analytic approximation for the results. We write the bispectra for matter and curvature perturbations, define a nonGaussianity parameter $f_{\mathrm{nl}}^{\sigma}$ for the model, and estimate the current constraint to $f_{\mathrm{nl}}^{\sigma}$ from the CMB. Section VI presents the matter bispectrum for modes that entered the horizon during radiation domination, those relevant for galaxy surveys. The central results of the paper are Eq. (33) for the curvature bispectrum; Eq. (32) which defines $f_{\mathrm{nl}}^{\sigma}$ in terms of the SOSF model parameters $v$ and $N$; Eq. (28) which approximates the bispectrum function $g_{3}\left(k_{1}, k_{2}, k_{3}\right)$; and Eqs. (34) and (35) which present the matter bispectrum in a form useful for galaxy surveys. We make concluding remarks in Sec. VII. An Appendix contains some calculational details and useful approximations.

\section{SCALAR-FIELD DYNAMICS}

Self-ordering scalar fields are described by an $N$-component scalar field with an $O(N)$ symmetry that is spontaneously broken to $O(N-1) \cdot{ }^{1}$ After symmetry breaking, the scalar field lies in different places in its $S^{N-1}$ vacuum manifold in different causally disconnected regions of the Universe. As the Universe expands and these previously causally disconnected regions come into causal contact, field gradients tend to align the scalar field. The rate of alignment for these fields is limited only by causality, and so the fields become aligned within a few Hubble times after horizon crossing. Still, as the Universe expands, there are continually new causally disconnected regions, on ever larger scales, that enter the horizon. The result is thus a continual scale-invariant generation of new scalar-field perturbations. In this section, we describe the scalar-field dynamics; the following section then describes how the gradient-energy density in these scalar fields induce perturbations to the matter density.

\footnotetext{
${ }^{1} \mathrm{We}$ assume that the issues about global symmetries raised in Refs. [25] are somehow solved [26].
}

The starting point is an $N$-component scalar field $\vec{\Phi}=$ $\left(\phi^{1}, \phi^{2}, \cdots, \phi^{N}\right)$, with $\phi^{a}$ real, with Lagrangian density,

$$
\mathcal{L}=-\left(\nabla_{\mu} \vec{\Phi}\right) \cdot\left(\nabla^{\mu} \vec{\Phi}\right)-\frac{\lambda}{4}\left(|\vec{\Phi}|^{2}-v^{2}\right)^{2}
$$

where $\lambda$ is the dimensionless self-coupling of $\vec{\Phi}$, and $v$ is the magnitude of the vacuum expectation value (vev) in the true vacuum. At temperatures $T \ll \lambda^{1 / 4} v$, the $O(N)$ symmetry of the Lagrangian is spontaneously broken, and the field is thereafter restricted to the $S^{N-1}$ vacuum manifold. The dynamics is thus effectively that of $N-1$ massless Nambu-Goldstone modes which we describe in terms of the $N$ fields $\phi^{a}$ with the effective Lagrangian density,

$$
\mathcal{L}=-\left(\nabla_{\mu} \vec{\Phi}\right) \cdot\left(\nabla^{\mu} \vec{\Phi}\right)+\Lambda\left(|\vec{\Phi}|^{2}-v^{2}\right),
$$

where $\Lambda$ is a Lagrange multiplier that enforces the constraint $|\vec{\Phi}|^{2}=v^{2}$. The resulting equations of motion are

$$
\begin{aligned}
\phi^{a \prime \prime}(\mathbf{x}, \eta) & +2 \mathcal{H} \phi^{a \prime}(\mathbf{x}, \eta) \\
& -\left[\nabla^{2}+\frac{1}{v^{2}}\left(\nabla_{\mu} \vec{\Phi}\right) \cdot\left(\nabla^{\mu} \vec{\Phi}\right)\right] \phi^{a}(\mathbf{x}, \eta)=0,
\end{aligned}
$$

where the primes denote derivatives with respect to conformal time $\eta$, and $\mathcal{H}=a^{\prime} / a$ in terms of the FriedmannRobertson-Walker scale factor $a(\eta)$. Also, $\nabla^{2}$ is here a spatial Laplacian in comoving coordinates. Equation (3) represents the nonlinear sigma model (NLSM from now on), that describes the evolution of the scalar field after spontaneous symmetry breaking.

In the large- $N$ limit, the field components become independent of each other (up to corrections of order $N^{-1}$ ). We thus replace the bilinear term in the equation of motion by an ensemble average,

$$
\left(\nabla_{\mu} \vec{\Phi}\right) \cdot\left(\nabla^{\mu} \vec{\Phi}\right)=N\left\langle\left(\nabla_{\mu} \phi^{a}\right)\left(\nabla^{\mu} \phi^{a}\right)\right\rangle \equiv T(\eta),
$$

where there is no sum on $a$ in the second equality, and in the last equality we have made the usual ergodic assumption, replacing the ensemble average by a spatial average.

The only time scale in the problem is that set by the (comoving) horizon $\mathcal{H}^{-1} \propto \eta$, so by dimensional considerations $T \propto \mathcal{H}^{2}$, and $T(\eta)=T_{o} / \eta^{2}$, with $T_{o}>0$. We then replace the nonlinear term in the NLSM equation of motion, Eq. (3), by this expectation value and in this way linearize the equations of motion. Introducing $\alpha=$ $d \log a / d \log \eta$ and Fourier transforming the spatial dependence of the equations,

$$
\phi^{a}(\mathbf{k}, \eta)=\int d^{3} x \phi^{a}(\mathbf{x}, \eta) e^{+i \mathbf{k} \cdot \mathbf{x}}
$$

we obtain

$$
\phi_{k}^{a^{\prime \prime}}+\frac{2 \alpha}{\eta} \phi_{k}^{a^{\prime}}+\left(k^{2}-\frac{T_{o}}{v^{2} \eta^{2}}\right) \phi_{k}^{a}=0,
$$

with $\alpha=1$ for a radiation-dominated Universe and $\alpha=2$ for a matter-dominated Universe. For constant $\alpha$, the so- 
lution to Eq. (6) that is finite as $\eta \rightarrow 0$, is $\phi^{a}(\mathbf{k}, \eta)=$ $\boldsymbol{v} \boldsymbol{\epsilon}^{a}(\mathbf{k}) f(k \eta)$, with $f(x) \equiv x^{1 / 2-\alpha} J_{\nu}(x)$, and $J_{\nu}(x)$ is a Bessel function. Here, $\boldsymbol{\epsilon}^{a}(\mathbf{k})$ is the amplitude of mode $\mathbf{k}$, and $\nu$ is fixed by $\nu^{2}=(1 / 2-\alpha)^{2}+\left(T_{o} / v^{2}\right)$.

In the large- $N$ limit, the statistical distribution of each field component approaches a Gaussian distribution with mean $\left\langle\phi^{a}(\mathbf{x}, \eta)\right\rangle=0$ and variance $\left\langle\phi^{a}(\mathbf{x}, \eta) \phi^{b}(\mathbf{x}, \eta)\right\rangle=$ $\left(v^{2} / N\right) \delta_{a b}$. The initial field component $\phi^{a}(\mathbf{x}, \eta=0)$ takes on a random value at each point in space. We thus take the $\left\{\epsilon^{a}(\mathbf{k})\right\}$ to be Gaussian random variables with mean $\left\langle\epsilon^{a}(\mathbf{k})\right\rangle=0$ and variance,

$$
\left\langle\epsilon^{a}(\mathbf{k}) \epsilon^{b}\left(\mathbf{k}^{\prime}\right)\right\rangle=(2 \pi)^{3}|\mathbf{k}|^{-n} \frac{\delta_{a b}}{A N} \delta_{D}\left(\mathbf{k}+\mathbf{k}^{\prime}\right),
$$

where $\delta_{D}(\mathbf{k})$ is the Dirac delta function, and $A$ is a normalization constant to be determined below. The powerlaw dependence on $k$ is taken since the initial conditions are scale-free.

The power-law index $n$ in Eq. (7) is fixed by the condition that $\left\langle\phi^{a}(\mathbf{x}, \eta) \phi^{b}(\mathbf{x}, \eta)\right\rangle=\left(v^{2} / N\right) \delta_{a b}$ for all $\eta$ :

$$
\begin{aligned}
\left\langle\phi^{a}(\mathbf{x}, \eta) \phi^{b}(\mathbf{x}, \eta)\right\rangle= & \delta_{a b} v^{2} \int \frac{d^{3} k}{(2 \pi)^{3}} \int \frac{d^{3} k^{\prime}}{(2 \pi)^{3}} \\
& \times\left\langle\epsilon^{a}(\mathbf{k}) \epsilon^{a}\left(\mathbf{k}^{\prime}\right)\right\rangle f(k \eta) f\left(k^{\prime} \eta\right) \\
= & \frac{4 \pi v^{2} \delta_{a b}}{(2 \pi)^{3} A N} \int d k k^{2-n} f^{2}(k \eta) .
\end{aligned}
$$

We see that $n=3$ gives a result that is independent of time, and so we choose $n=3$ hereafter.

Just after symmetry breaking, at conformal time $\eta_{*}$, the field correlation is then

$$
\begin{aligned}
\left\langle\phi^{a}\left(\mathbf{k}, \eta_{*}\right) \phi^{b}\left(\mathbf{k}^{\prime}, \eta_{*}\right)\right\rangle & \propto f^{2}\left(x_{*}\right)\left\langle\epsilon^{a}(\mathbf{k}) \epsilon^{b}\left(\mathbf{k}^{\prime}\right)\right\rangle \\
& \propto \eta_{*}^{3}|\mathbf{k}|^{1-2 \alpha+2 \nu-3} \delta_{D}\left(\mathbf{k}+\mathbf{k}^{\prime}\right) .
\end{aligned}
$$

Since the initial values $\phi^{a}\left(\mathbf{x}, \eta_{*}\right)$ are uncorrelated on scales $k \ll \eta_{*}^{-1}$, we set $\nu=\alpha+1$, so that the initial field is described by a white-noise power spectrum. This then fixes $\left(T_{o} / v^{2}\right)=3 \alpha+(3 / 4)$.

We now return to Eq. (8) to fix the normalization constant $A$. From

$$
\begin{aligned}
\left\langle\phi^{a}(\mathbf{x}, \eta) \phi^{b}(\mathbf{x}, \eta)\right\rangle & =\frac{v^{2} \delta_{a b}}{2 \pi^{2} A N} \int_{0}^{\infty} d x x^{-2 \alpha} J_{\alpha+1}^{2}(x) \\
& =\frac{v^{2} \delta_{a b}}{N}
\end{aligned}
$$

we find

$$
A=\frac{1}{8 \pi^{2}} \frac{\Gamma(\alpha)}{\Gamma(2 \alpha+3 / 2) \Gamma(\alpha+1 / 2)} .
$$

For $\alpha=2$ (matter domination), $\quad A=16 / 2835 \pi^{3}=$ $1.82 \times 10^{-4}$, and for $\alpha=1$ (radiation domination), $A=$ $2 / 15 \pi^{3}=4.3 \times 10^{-3}$.

\section{MATTER-DENSITY PERTURBATIONS}

Although the scalar field will initially take on different values in different causally disconnected regions, the curvature perturbation is initially zero. The scalar-field gradient-energy perturbation that arises as previously causally disconnected regions come into causal contact is then compensated by a perturbation in the matter density [23,27].

In this section, we calculate the time evolution of the matter perturbation. The action of the scalar field occurs primarily within a few Hubble times after a particular Fourier mode $k$ enters the horizon. The subsequent evolution of the mode is then governed by gravitational infall as if it were a primordial perturbation; i.e., the perturbation amplitude grows only logarithmically during radiation domination, and then grows with the scale factor during matter domination. Our strategy here will be to evaluate the matter-perturbation amplitude several Hubble times after horizon crossing, a calculation that is relatively straightforward. Strictly speaking, our calculation applies only to modes that enter the horizon during matter domination, but we argue below that our ultimate results for the bispectrum should also be roughly valid for the smaller-scale modes that enter the horizon during radiation domination, those relevant for galaxy surveys.

As described in Ref. [23], the scalar-field alignment involves density perturbations that then lead to gravitational-potential perturbations which in turn induce the perturbations to the matter density that are our ultimate interest. Following Ref. [23], the matter-density perturbation induced by the scalar field for modes that enter the horizon during matter domination is

$$
\delta(\mathbf{x}, \eta)=\frac{2 \pi G}{5} \eta^{2} \int d \eta^{\prime} \partial_{i} T_{0 i}\left(\mathbf{x}, \eta^{\prime}\right)
$$

where $G$ is Newton's constant, and

$$
T_{0 i}=\left(\partial_{0} \phi^{a}\right)\left(\partial_{i} \phi^{a}\right),
$$

is the $0 i$-component of the stress-energy tensor of the multicomponent scalar field. The integral in Eq. (12) approaches a constant for $\eta \gg$ few $/ k$-i.e., within a few Hubble times after horizon crossing. The subsequent $\eta^{2}$ evolution in the prefactor is then simply the $\delta \propto a \propto \eta^{2}$ linear-theory growth of the perturbation amplitude in a matter-dominated Universe.

Using $G=1 / M_{\mathrm{Pl}}^{2}$, where $M_{\mathrm{Pl}}=1.22 \times 10^{19} \mathrm{GeV}$ is the Planck mass, and defining

$$
C \equiv \frac{2 \pi}{5}\left(\frac{v}{M_{\mathrm{Pl}}}\right)^{2}
$$

the Fourier transform of the density perturbation is 


$$
\begin{aligned}
\delta(\mathbf{k}, \eta)= & -C \eta^{2} \int \frac{d^{3} q}{(2 \pi)^{3}} \epsilon^{a}(\mathbf{q}) \epsilon^{a}(\mathbf{k}-\mathbf{q})|\mathbf{k}-\mathbf{q}|(\mathbf{k} \cdot \mathbf{q}) \\
& \times \int d \tau f^{\prime}(|\mathbf{k}-\mathbf{q}| \tau) f(q \tau)
\end{aligned}
$$

where $f^{\prime}(y) \equiv d f / d y$. The crucial qualitative feature is that $\delta(\mathbf{k}, \eta)$ is quadratic in powers of $\epsilon^{a}(\mathbf{k})$. And since $\epsilon^{a}(\mathbf{k})$ is a nearly Gaussian field, the density field $\delta(\mathbf{x})$ will be highly non-Gaussian.

\section{THE POWER SPECTRUM}

The power spectrum $P^{\sigma}(k)$ for matter-density perturbations induced by the scalar field is defined by

$$
\left\langle\delta(\mathbf{k}) \delta\left(\mathbf{k}^{\prime}\right)\right\rangle=(2 \pi)^{3} \delta_{D}\left(\mathbf{k}+\mathbf{k}^{\prime}\right) P^{\sigma}(k),
$$

where the angle brackets denote an average over all realizations of the random field $\delta(\mathbf{k})$. The calculation of the power spectrum is lengthy but straightforward; details are provided in the Appendix. The result, given in Eq. (A5), can be rewritten,

$$
\begin{aligned}
P^{\sigma}(k, \eta) \equiv & \frac{C^{2} \eta^{4}}{(2 \pi)^{2} A^{2}} \frac{k}{N} \int_{0}^{\infty} d v v^{3} \int_{-1}^{1} d l I(v, b) \\
& \times l[I(v, b) v l+I(b, v)(1-v l)],
\end{aligned}
$$

where $b=\sqrt{1+v^{2}-2 v l}$, and

$$
I(a, b) \equiv \int d s \frac{f(a s) f^{\prime}(b s)}{a^{3 / 2} b^{1 / 2}} .
$$

Strictly speaking, the upper limit in this integral is $k \eta$. However, here we will restrict our attention to modes that have evolved well within the horizon, $k \eta \gg 1$, and so we take the upper limit of the integral in Eq. (18) to be infinity. In this case, the integral $I(a, b)$ is antisymmetric in its arguments, and the power spectrum can be written

$$
P^{\sigma}(k, \eta) \equiv \frac{C^{2} \eta^{4}}{A^{2}} \frac{k}{N} g_{2},
$$

where

$$
g_{2} \equiv \int \frac{d^{3} v}{(2 \pi)^{3}}[I(v,|\hat{\mathbf{z}}-\mathbf{v}|)]^{2}(\hat{\mathbf{z}} \cdot \mathbf{v})[2(\hat{\mathbf{z}} \cdot \mathbf{v})-1],
$$

and $\hat{\mathbf{z}}$ is a unit vector. Details on the evaluation of $I$ are given in the Appendix. For $\alpha=2$ (matter domination), the integral evaluates to $g_{2}=3.3 \times 10^{-7}$ and for $\alpha=1$ (radiation domination) it is $g_{2}=2.1 \times 10^{-4}$. Note that the ratios $g_{2} / A^{2}$ that appear in Eq. (20) are approximately 10 and 11, respectively, for $\alpha=2,1$, implying that the amplitude of the matter perturbation induced by the unwinding of the scalar field is the same, to $O(10 \%)$, for modes that enter the horizon during matter and radiation domination.

\section{Normalization of the power spectrum}

We now estimate the constraints to the $v-N$ parameter space from the empirical constraint that the SOSF provide no more than a fraction $p_{\sigma} \simeq 0.1$ to $C_{l=10}$ [8], the CMB temperature power spectrum at multipole moment $l=10$.

On subhorizon scales during matter domination, the curvature perturbation $\zeta(\mathbf{x})$ is related to the gravitational potential $\Phi(\mathbf{x})$ by $\zeta(\mathbf{x})=(5 / 3) \Phi(\mathbf{x})$. The gravitational potential is related to the density perturbation through the Poisson equation, $\nabla^{2} \Phi=4 \pi G a^{2} \bar{\rho} \delta$, where $\bar{\rho}$ is the mean density. In Fourier space, the curvature perturbation $\zeta(\mathbf{k})$ is thus related to the matter-density perturbation $\delta(\mathbf{k})$ by

$$
\zeta(\mathbf{k})=-\frac{5}{2}\left(\frac{a H}{k}\right)^{2} \delta(\mathbf{k}),
$$

where we have used the Friedmann equation $H^{2}=$ $8 \pi G \rho / 3$, and $H=(d a / d t) / a$ is the expansion rate. The amplitude of the curvature power spectrum due to the SOSF is therefore,

$$
\begin{aligned}
\Delta_{\mathcal{R} \sigma}^{2} & \equiv \frac{k^{3}}{2 \pi^{2}} P_{\zeta}(k)=\frac{k^{3}}{2 \pi^{2}}\left[\frac{5}{2}\left(\frac{a H}{k}\right)^{2}\right]^{2} P(k) \\
& =8\left(\frac{v}{M_{\mathrm{Pl}}}\right)^{4} \frac{g_{2}}{A^{2} N} \simeq 80\left(\frac{v}{M_{\mathrm{Pl}}}\right)^{4} \frac{1}{N},
\end{aligned}
$$

where we have used $(a H \eta)=2$ during matter domination. The next step is then to determine the relation between the curvature-power-spectrum amplitude $\Delta_{\mathcal{R} \sigma}^{2}$ and the temperature-fluctuation amplitude. This is a notoriously difficult calculation, but to get an estimate, we use Fig. 4 in Ref. [28], which shows that the large-angle temperature fluctuation $\Delta T$ in a SOSF model is $G_{\mathrm{sw}} \simeq 10$ times greater than it would be in an adiabatic model with the same matter-power-spectrum normalization on large scales. ${ }^{2}$ Current CMB measurements indicate a curvature power spectrum $\Delta_{\mathcal{R}} \simeq 5 \times 10^{-5}$, if primordial perturbations are adiabatic. If the SOSF provides $G_{\mathrm{sw}}$ times more $\Delta T$ for fixed curvature, and if they make a fractional contribution $p_{\sigma}$ to the large-angle temperature variance, then $\Delta_{\mathcal{R} \sigma}^{2}=$ $\left(p_{\sigma} / G_{\mathrm{sw}}^{2}\right) \Delta_{\mathcal{R}}^{2}$. We thus obtain

$$
\frac{v}{N^{1 / 4}}=\left(\frac{p_{\sigma} A^{2} \Delta_{\mathcal{R}}^{2}}{8 G_{\mathrm{sw}}^{2} g_{2}}\right)^{1 / 4} M_{\mathrm{Pl}} \lesssim \frac{M_{\mathrm{Pl}}}{2000},
$$

where the numerical result is obtained by taking $p_{\sigma}=0.1$ and $G_{\mathrm{sw}}=10$. The numerical upper limit in Eq. (23) is in good agreement with limits obtained from simulations [29].

\footnotetext{
${ }^{2}$ The factor of 10 is a bit larger than the factor of 6 one might attribute due to the difference (1/3 versus 2$)$ for the Sachs-Wolfe amplitude for adiabatic and isocurvature perturbations. The additional $\Delta T$ may be due in part to the vector and tensor perturbations that are also excited in SOSF models.
} 


\section{THE BISPECTRUM}

\section{A. The calculation}

The calculation of the bispectrum proceeds analogously. The matter bispectrum $B\left(k_{1}, k_{2}, k_{3}\right)$ is defined by

$\left\langle\delta\left(\mathbf{k}_{1}\right) \delta\left(\mathbf{k}_{2}\right) \delta\left(\mathbf{k}_{3}\right)\right\rangle=(2 \pi)^{3} \delta_{D}\left(\mathbf{k}_{1}+\mathbf{k}_{2}+\mathbf{k}_{3}\right) B\left(k_{1}, k_{2}, k_{3}\right)$.

Although the definition of the bispectrum is nominally in terms of the vector quantities $\mathbf{k}_{i}$, the triangle constraint $\mathbf{k}_{1}+\mathbf{k}_{2}+\mathbf{k}_{3}=0$ imposed by the Dirac delta function, as well as statistical isotropy, imply that the bispectrum is most generally a function of the magnitudes $k_{i}$ of the three sides of the triangle. Again, some details of the calculation are provided in the Appendix. The result is

$$
B\left(k_{1}, k_{2}, k_{3}\right)=\frac{C^{3} \eta^{6}}{A^{3} N^{2}} g_{3}\left(k_{1}, k_{2}, k_{3}\right),
$$

where

$$
\begin{aligned}
g_{3}\left(k_{1}, k_{2}, k_{3}\right) \equiv & \int \frac{d^{3} v}{(2 \pi)^{3}} H(\mathbf{u}+\mathbf{v}, \mathbf{v}) H(\mathbf{v}, \hat{\mathbf{z}}-\mathbf{v}) \\
& \times H(\hat{\mathbf{z}}-\mathbf{v}, \mathbf{u}+\mathbf{v}),
\end{aligned}
$$

with

$$
H(\mathbf{a}, \mathbf{b}) \equiv I(a, b)\left(b^{2}-a^{2}\right) .
$$

We have chosen $\vec{k}_{1}$ in Eq. (26) to be in the $\hat{\mathbf{z}}$ direction, without loss of generality, and we have then defined $\mathbf{u} \equiv$ $\mathbf{k}_{2} / k_{1}$. Note that $H(\vec{a}, \vec{b})=H(a, b)=H(b, a)$; i.e., it is a function only of the magnitudes of its arguments, and it is symmetric in its arguments. Note further that $H(a, b) \leq 0$, and thus $g_{3}\left(k_{1}, k_{2}, k_{3}\right)<0$. The function $g_{3}\left(k_{1}, k_{2}, k_{3}\right)$ depends only on the shape of the triangle, not on its overall size-i.e., $g_{3}\left(k_{1}, k_{2}, k_{3}\right)=g_{3}\left(1, k_{2} / k_{1}, k_{3} / k_{1}\right)$-a consequence of the scale invariance of SOSFs. We have checked that Eq. (26) is equivalent to, although far simpler, than Eq. (59) in Ref. [23]. Given the symmetry of $H(a, b)$ in its arguments, it is simple to check that $g_{3}(\mathbf{w}-\hat{\mathbf{z}}-\mathbf{u})=$ $g(\mathbf{u})$, as it should (given that the three sides of the triangle should add as $\hat{\mathbf{z}}+\mathbf{u}+\mathbf{w}=0$ ). If we set the third side to have length $w=k_{3} / k_{1}$, then $\cos \theta \equiv \mathbf{u} \cdot \hat{\mathbf{z}}=\left(w^{2}-1-\right.$ $\left.u^{2}\right) /(2 u)$. If we choose $k_{1} \geq k_{2} \geq k_{3}$, then $\cos \theta<$ $-(2 u)^{-1}$.

We have calculated $g_{3}\left(k_{1}, k_{2}, k_{3}\right)$ numerically, and the result is shown in Fig. 1. We note (prefacing the discussion below) that the quantity, $-g_{3}\left(1, x_{2}, x_{3}\right)$, with $x_{2}=k_{2} / k_{1}$ and $x_{3}=k_{3} / k_{1}$, that we plot is the same (up to some normalization factor) as the quantity $F\left(q, x_{2}, x_{3}\right) x_{2}^{2} x_{3}^{2}$ plotted in Figs. 1 and 2 in Ref. [30] which show, respectively, the bispectra for the local model and equilateral model. Those figures show that the local-model bispectrum peaks sharply for "squeezed" triangles $\left(k_{1} \simeq k_{2} \gg k_{3}\right)$ and that the equilateral-model bispectrum peaks at equilateral triangles $\left(k_{1} \simeq k_{2} \simeq k_{3}\right)$. Our Fig. 1 shows that the SOSF

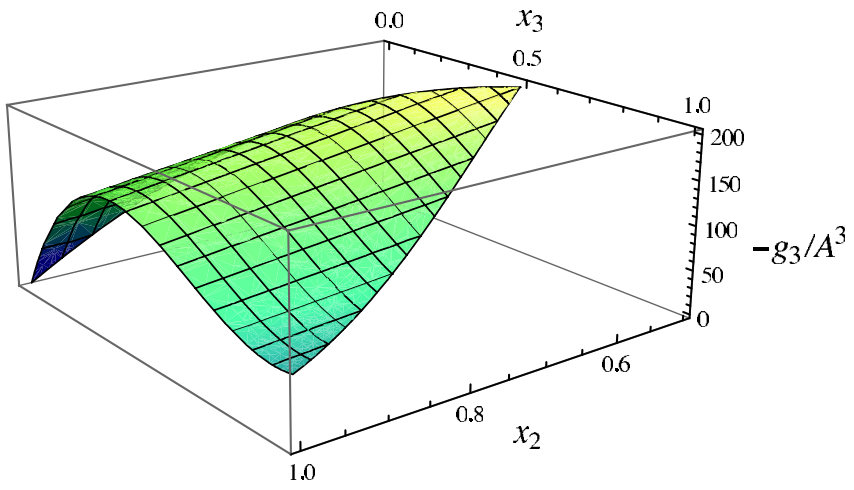

FIG. 1 (color online). The function $-g_{3}\left(k_{1}, k_{2}, k_{3}\right)$, taking $k_{1}=1$, for modes that enter the horizon during matter domination. The figure looks virtually identical for modes that enter the horizon during radiation domination.

bispectrum is, however, quite different. It is nonzero for equilateral triangles, goes to zero in the squeezed limit, and it peaks for "aligned" triangles, $k_{1} \simeq 2 k_{2} \simeq 2 k_{3}$.

To aid in data-analysis efforts, we have found that the following approximation reproduces the numerical results for $g_{3}\left(k_{1}, k_{2}, k_{3}\right)$ to within a few percent:

$$
\begin{aligned}
g_{3}\left(k_{1}, k_{2}, k_{3}\right)= & -\frac{A^{3}}{143}\left(262-127 \frac{k_{2}}{k_{1}}\right) \\
& \times\left[947 \frac{k_{3}}{k_{1}}-1770\left(\frac{k_{3}}{k_{1}}\right)^{2}+893\left(\frac{k_{3}}{k_{1}}\right)^{3}\right],
\end{aligned}
$$

where we take $k_{1} \geq k_{2} \geq k_{3}$ in this expression.

\section{B. Curvature Bispectrum}

To compare with results for other models, and for comparison with $\mathrm{CMB}$ constraints, we next calculate the curvature bispectrum $F\left(k_{1}, k_{2}, k_{3}\right)$, defined by

$\left\langle\zeta\left(\mathbf{k}_{1}\right) \zeta\left(\mathbf{k}_{2}\right) \zeta\left(\mathbf{k}_{3}\right)\right\rangle=(2 \pi)^{3} \delta_{D}\left(\mathbf{k}_{1}+\mathbf{k}_{2}+\mathbf{k}_{3}\right) F\left(k_{1}, k_{2}, k_{3}\right)$.

Using the relations above, we can write $C^{2}=\left(\pi^{2} / 50\right) \times$ $\left(A^{2} N \Delta_{\mathcal{R} \sigma}^{2} / g_{2}\right)$ and then find,

$$
F^{\sigma}\left(k_{1}, k_{2}, k_{3}\right)=-\frac{2 \sqrt{2} \pi^{3} \Delta_{\mathcal{R} \sigma}^{3}}{g_{2}^{3 / 2} N^{1 / 2}} \frac{g_{3}\left(k_{1}, k_{2}, k_{3}\right)}{k_{1}^{2} k_{2}^{2} k_{3}^{2}} .
$$

A few observations: (1) It is only the amplitude, not the shape, that depends on the symmetry-breaking scale $v$. (2) The bispectrum decreases, for fixed $\Delta_{\mathcal{R} \sigma}$, as $N^{-1 / 2}$ with increasing $N$, again reflecting that the model should become increasingly Gaussian with more fields, a consequence of the central-limit theorem. (3) The scaling with $\Delta_{\mathcal{R}}$ is $\propto \Delta_{\mathcal{R}}^{3}$, as opposed to the $\Delta_{\mathcal{R}}^{4}$ scaling of the localmodel bispectrum. In words, the non-Gaussianity is of order unity, a consequence of the fact that the density 
perturbation is the square of a Gaussian random field [cf., Eq. (12)], rather than something very small, as in inflationary models.

We now put the curvature bispectrum in a slightly more familiar form by defining a non-Gaussianity parameter $f_{\mathrm{nl}}^{\sigma}$ for the model. To do so, we recall that the local-model prediction for the curvature bispectrum is

$$
\begin{aligned}
F^{\text {local }}\left(k_{1}, k_{2}, k_{3}\right)= & 2 \frac{3}{5}\left(2 \pi^{2}\right)^{2} \Delta_{\mathcal{R}}^{4} f_{\mathrm{nl}}^{\text {local }} \\
& \times\left[\frac{1}{k_{1}^{3} k_{2}^{3}}+\frac{1}{k_{2}^{3} k_{3}^{3}}+\frac{1}{k_{1}^{3} k_{3}^{3}}\right],
\end{aligned}
$$

where $f_{\mathrm{nl}}^{\text {local }}$ is the local-model non-Gaussianity parameter, defined by writing the curvature as $\zeta(\mathbf{x})=\zeta_{g}(\mathbf{x})+$ $(3 / 5) f_{\mathrm{nl}}^{\text {local }}\left[\left(\zeta_{g}\right)^{2}(\mathbf{x})-\left\langle\left(\zeta_{g}\right)^{2}(\mathbf{x})\right\rangle\right]$ in terms of a Gaussian field $\zeta_{g}(\mathbf{x})$.

We now define the non-Gaussianity parameter $f_{\mathrm{nl}}^{\sigma}$ for SOSFs by equating the local-model and SOSF bispectra for equilateral triangles; i.e., equating Eqs. (31) and (30), we define,

$$
\begin{aligned}
f_{\mathrm{nl}}^{\sigma} & \equiv-\frac{5 p_{\sigma}^{3 / 2} g_{3}(1,1,1)}{18 \sqrt{2} \pi N^{1 / 2} g_{2}^{3 / 2} \Delta_{\mathcal{R}} G_{\mathrm{sw}}^{3}} \\
& \simeq 40 G_{\mathrm{sw}}^{-3}\left(\frac{p_{\sigma}}{0.1}\right)^{3 / 2}\left(\frac{N}{5}\right)^{-1 / 2} \\
& \simeq 3 G_{\mathrm{sw}}^{-3}\left(\frac{v}{5 \times 10^{15} \mathrm{GeV}}\right)^{6}\left(\frac{N}{5}\right)^{-2} .
\end{aligned}
$$

With this $f_{\mathrm{nl}}^{\sigma}$, the curvature bispectrum can then be written

$$
F\left(k_{1}, k_{2}, k_{3}\right)=\frac{18}{5}\left(2 \pi^{2}\right)^{2} \Delta_{\mathcal{R}}^{4} \frac{f_{\mathrm{nl}}^{\sigma}}{k_{1}^{2} k_{2}^{2} k_{3}^{2}} \frac{g_{3}\left(k_{1}, k_{2}, k_{3}\right)}{g_{3}(1,1,1)} .
$$

Similarly, the gravitational-potential bispectrum can be obtained by multiplying this expression by $5 / 3$ and then replacing $\Delta_{\mathcal{R}}^{2}$ by $\Delta_{\Phi}^{2}=(3 / 5)^{2} \Delta_{\mathcal{R}}^{2}$. Note that $f_{\mathrm{nl}}^{\sigma}$ is manifestly positive, unlike $f_{\mathrm{nl}}$ for the local or equilateral models, which may take on either sign.

\section{Estimate of CMB Constraints}

As indicated in the Introduction, the bispectrum can be probed with the CMB, large-scale structure, and the abundance of objects. The strongest current constraints to the local-model bispectrum come from the CMB [31], followed closely by galaxy-clustering constraints. Given that SOSFs produce a larger temperature-fluctuation amplitude for a given density-perturbation amplitude, we surmise that the $\mathrm{CMB}$ will provide stronger constraints to SOSF non-Gaussianity than galaxy clustering. We thus now estimate a constraint to $f_{\mathrm{nl}}^{\sigma}$ from the CMB.

Before doing so, we first caution that Eq. (33) is derived for the curvature perturbation only for modes once they are well within the horizon. It is thus not, strictly speaking, appropriate for CMB modes $l \lesssim 100$. Still, the shape dependence of the bispectrum, and its amplitude relative to the curvature-perturbation amplitude, arises primarily from the quadratic dependence of the density perturbation on the scalar-field perturbation as encoded in Eq. (15). The shape dependence of the bispectrum we calculate should thus be at least roughly correct even for $l \lesssim 100$.

CMB constraints to $f_{\mathrm{nl}}$ are typically applied assuming that the density perturbations are adiabatic, which implies a certain relation, $(\Delta T / T) \simeq-\zeta / 5$, for the large-angle temperature fluctuation. In our case, though, there is roughly $G_{\mathrm{sw}} \simeq 10$ times more $\Delta T$ for a given $\zeta$ than in adiabatic models. If so, and if all of these temperature fluctuations are due to scalar perturbations (rather than vector and/or tensor modes), then the implied CMB bispectrum should be roughly $G_{s w}^{3}$ times larger. Simulations show, though, that only a fraction $f_{\mathrm{s}} \simeq 0.5$ of the SOSF temperature-fluctuation power is due to scalar modes, the rest coming from vector and tensor perturbations $[28,32]$. The implied CMB bispectrum should thus scale with $f_{\mathrm{s}}^{3 / 2}$. Combining these scalings with $f_{\mathrm{nl}}^{\sigma} \propto G_{\mathrm{sw}}^{-3}$ [see Eq. (32)], the $G_{\mathrm{sw}}$ dependence of the CMB bispectrum drops out. We can therefore apply CMB constraints to $f_{\mathrm{nl}}^{\sigma}$ by identifying the $f_{\mathrm{nl}}$ constraints obtained from the CMB for adiabatic perturbations with $G_{\mathrm{sw}}^{3} f_{\mathrm{s}}^{3 / 2} f_{\mathrm{nl}}^{\sigma}$. And one final caveat: We disregard the differences in the temperature power spectra from SOSFs and adiabatic perturbations.

Keeping these multiple caveats in mind, we proceed with our very rough estimate by noting that the WMAP-7 95\% C.L. limit to $f_{\mathrm{nl}}^{\text {equil }}$, the non-Gaussianity parameter for the equilateral model, is $-211<f_{\mathrm{nl}}^{\text {equil }}<266$ [33]; this bispectrum is maximized for equilateral triangles. On the other hand, the SOSF bispectrum is maximized for aligned triangles and is zero for squeezed triangles. We thus conclude that the constraint to $G_{\mathrm{sw}}^{3} f_{\mathrm{s}}^{3 / 2} f_{\mathrm{nl}}^{\sigma}$ will be stronger than that to $f_{\mathrm{nl}}^{\text {equil }}$, but it is not clear-given the different weightings to squeezed and aligned triangles-how it will compare with that to $f_{\mathrm{nl}}^{\text {local }}$. Applying these rough arguments to Eq. (32), with $f_{\mathrm{s}} \simeq 0.5$, then we estimate a non-Gaussianity parameter in excess of the predicted threshold $f_{\mathrm{nl}} \sim 7$ for detection by Planck [34]. For now, however, we simply estimate conservatively that $\left|G_{\mathrm{sw}}^{3} f_{\mathrm{s}}^{3 / 2} f_{\mathrm{nl}}^{\sigma}\right| \lesssim 200$.

\section{THE BISPECTRUM FOR GALAXY SURVEYS}

We have carried out our calculations in the regime where analytic progress is most easily made-i.e., modes that have entered the horizon during matter domination and only after those modes have evolved well within the horizon. Strictly speaking, therefore, our calculations apply only to galaxy surveys on very large scales-those generally larger than extant surveys cover-and possibly to midscale regimes in the CMB.

Still, our results for the bispectrum can be easily adapted to obtain roughly the bispectrum for smaller-scale modes, those that enter the horizon during radiation domination and those relevant for galaxy surveys. The calculation for 
the evolution of these modes is altered by three effects: (1) The index $\nu=1+\alpha$ for the Bessel functions in the scalar-field dynamics (Sec. II) is different. We have already shown that this has no more than an $O(10 \%)$ effect on the power spectrum. We have also calculated $g_{3}\left(k_{1}, k_{2}, k_{3}\right)$ for modes that enter the horizon during radiation domination. We find that the combination $g_{3}(1,1,1) / g_{2}^{3 / 2}$ that appears in the bispectrum amplitude [see Eq. (34) below] is bigger by $10 \%$ for modes that enter the horizon during radiation domination than for those that enter during matter domination. We also find that the shape dependence $g_{3}\left(k_{1}, k_{2}, k_{3}\right) / g_{3}(1,1,1$,$) is similar at the O(10 \%)$ level for modes that enter the horizon during matter and radiation domination. This is simply because the correlations between different Fourier modes of the scalar-field energy density are imprinted, through Eq. (15), by the dependence of those Fourier modes on the scalar-field perturbations. This is the same for modes that enter the horizon during matter and radiation domination. The other two effects are (2) a slightly different amplitude for the matter perturbation, relative to the scalar-field energy-density perturbation, for modes that enter the horizon during radiation domination [Eq. (32) for radiation domination in Ref. [23], as opposed to Eq. (29) in the same reference, our Eq. (12), for matter domination]; and (3) the usual linear-theory growth of primordial isocurvature perturbations through radiation domination and the transition to matter domination. These latter two effects amount to a calculation of the transfer function $T(k)$ for the matter power spectrum in SOSF models, which can be accomplished either with simulations [cf., Refs. [28,29,35]] or approximately with standard linear-theory calculations with primordial isocurvature fluctuations. Again, however, although the calculation of the evolution of the amplitudes of the small-scale density-perturbation Fourier modes will be far more complicated than the larger-scale modes we have focused upon, the correlations between those modes will be, at the $O(10 \%)$ level, the same as those we have calculated for larger-scale modes.

More precisely, all we need to do is replace the density fields $\delta(\mathbf{k})$ in Secs. IV and VA by $\delta(\mathbf{k}) T(k)$, where $T(k)$ is the SOSF transfer function. The matter power spectrum $P^{\sigma}(k)$ due to SOSFs is then obtained from that in Eq. (19) by multiplying by $|T(k)|^{2}$, and the matter bispectrum is obtained by multiplying that in Eq. (25) by $T\left(k_{1}\right) T\left(k_{2}\right) T\left(k_{3}\right)$. We can then write the normalization constant $\left(C \eta^{2} / A\right)^{3}$ in Eq. (25) in terms of the (processed) matter power spectra using Eq. (19) to obtain the matter bispectrum,

$$
B\left(k_{1}, k_{2}, k_{3}\right)=\frac{g_{3}\left(k_{1}, k_{2}, k_{3}\right)}{g_{2}^{3 / 2} N^{1 / 2}}\left[\frac{P^{\sigma}\left(k_{1}\right) P^{\sigma}\left(k_{2}\right) P^{\sigma}\left(k_{3}\right)}{k_{1} k_{2} k_{3}}\right]^{1 / 2},
$$

valid for galaxy-survey scales. Here $P^{\sigma}(k)$ is the processed power spectrum due to SOSFs; i.e., it includes the transfer function. Using Eq. (32), this can be rewritten in terms of $f_{\mathrm{nl}}^{\sigma}$ as,

$$
\begin{aligned}
B\left(k_{1}, k_{2}, k_{3}\right)= & \frac{18 \sqrt{2} \pi f_{\mathrm{nl}}^{\sigma} \Delta_{\mathcal{R}} G_{\mathrm{sw}}^{3}}{5 p_{\sigma}^{3 / 2}} \frac{g_{3}\left(k_{1}, k_{2}, k_{3}\right)}{g_{3}(1,1,1)} \\
& \times\left[\frac{P^{\sigma}\left(k_{1}\right) P^{\sigma}\left(k_{2}\right) P^{\sigma}\left(k_{3}\right)}{k_{1} k_{2} k_{3}}\right]^{1 / 2} \\
\simeq & 25 f_{\mathrm{nl}}^{\sigma}\left(\frac{G_{\mathrm{sw}}}{10}\right)^{3}\left(\frac{p_{\sigma}}{0.1}\right)^{-3 / 2} \frac{g_{3}\left(k_{1}, k_{2}, k_{3}\right)}{g_{3}(1,1,1)} \\
& \times\left[\frac{P^{\sigma}\left(k_{1}\right) P^{\sigma}\left(k_{2}\right) P^{\sigma}\left(k_{3}\right)}{k_{1} k_{2} k_{3}}\right]^{1 / 2} .
\end{aligned}
$$

We leave further evaluation of this bispectrum, as well as assessment of current constraints, for future work.

\section{DISCUSSION}

If some post-inflationary physics involves the spontaneous breaking of an exact $O(N)$ symmetry with $N>4$, then the ordering of these scalar fields may provide a secondary contribution to primordial perturbations. Current constraints allow up to $\sim 10 \%$ of the power in primordial perturbations to be due to SOSFs. SOSF models are appealing from the theoretical perspective because they are simple, well defined, and parametrized only by the symmetry-breaking scale $v$ and number $N$ of fields.

In this paper we have calculated the matter and curvature bispectra induced by the ordering of such scalar fields. Given that the density perturbation is quadratic in the scalar-field perturbation, SOSF density perturbations are expected to be highly non-Gaussian, and if so, measurements of non-Gaussianity may provide the means to test these models.

Here we have calculated analytically the bispectrum due to SOSFs and presented results in a way that should be easily accessible to those doing measurements with the CMB and large-scale structure. We find that the triangleshape dependence of the bispectrum peaks for aligned triangles, unlike the local-model bispectrum, which is largest for squeezed triangles, and the equilateral bispectrum, which is largest for equilateral triangles. We have estimated a current upper limit to the non-Gaussianity parameter $f_{\mathrm{nl}}^{\sigma}$ for the model and find that the implied constraints to the $v$ - $N$ SOSF parameter space are competitive with those from the upper limit to CMB temperature fluctuations.

Finally, we have already argued above, in Sec. V C, that the correlation of modes will be similar for the large-scale modes as they enter the horizon, those relevant for largeangle CMB fluctuations. We therefore believe that rough constraints to the model can be derived from CMB measurements by assuming that the curvature bispectrum we calculate is the primordial one.

Clearly, there is room for further numerical work to test our assumptions and to make our predictions more precise. 
In the meantime, though, we believe that our analytic approximation captures the essential physics and that our bispectrum can be used in the meantime as a "workinghorse" model to derive constraints, from non-Gaussianity measurements, to this interesting class of models for secondary contributions to primordial perturbations.

Finally, we note that the model makes a number of other predictions. Given that density perturbations are actively generated as new modes come within the horizon, vector and tensor modes will be excited, and these may give rise to interesting polarization signals [36] in the CMB and perhaps excite B modes [37] in the CMB that might be distinguished from those due to inflation [38]. There will also be a scale-invariant spectrum of primordial gravitational waves produced [39] that can be sought in gravitational-wave observatories.

\section{ACKNOWLEDGMENTS}

DGF thanks Caltech and CERN for hospitality during the completion of this work. DGF was supported by the Spanish Ministry of Science under Contract No. AP-20051092. This work was supported at Caltech by DOE under Contract No. DE-FG03-92-ER40701 and the Gordon and Betty Moore Foundation, and at Dartmouth by NSF under Contract No. AST-0349213.

\section{APPENDIX: CALCULATIONAL DETAILS}

\section{The power spectrum}

From Eq. (15), the two-point correlator in Eq. (16) is expressed in terms of the correlator of four $\epsilon^{a}(\mathbf{k})$ 's. Since $\epsilon^{a}(\mathbf{k})$ 's are Gaussian distributed, we find via the Wick theorem,

$$
\begin{aligned}
\left\langle\delta(\mathbf{k}) \delta\left(\mathbf{k}^{\prime}\right)\right\rangle= & C^{2} \eta^{4} \int \frac{d^{3} q d^{3} q^{\prime}}{(2 \pi)^{6}}\left\langle\epsilon^{a}(\mathbf{q}) \boldsymbol{\epsilon}^{a}(\mathbf{k}-\mathbf{q}) \boldsymbol{\epsilon}^{b}\left(\mathbf{q}^{\prime}\right) \boldsymbol{\epsilon}^{b}\left(\mathbf{k}^{\prime}-\mathbf{q}^{\prime}\right)\right\rangle|\mathbf{k}-\mathbf{q}|\left|\mathbf{k}^{\prime}-\mathbf{q}^{\prime}\right|(\mathbf{k} \cdot \mathbf{q})\left(\mathbf{k}^{\prime} \cdot \mathbf{q}^{\prime}\right) \\
& \times \int d \tau f^{\prime}(|\mathbf{k}-\mathbf{q}| \tau) f(q \tau) \int d \xi f^{\prime}\left(\left|\mathbf{k}^{\prime}-\mathbf{q}^{\prime}\right| \xi\right) f\left(q^{\prime} \xi\right) \\
= & C^{2} \eta^{4} \int \frac{d^{3} q d^{3} q^{\prime}}{(2 \pi)^{6}}\left[\left\langle\epsilon^{a}(\mathbf{q}) \epsilon^{a}(\mathbf{k}-\mathbf{q})\right\rangle\left\langle\epsilon^{b}\left(\mathbf{q}^{\prime}\right) \boldsymbol{\epsilon}^{b}\left(\mathbf{k}^{\prime}-\mathbf{q}^{\prime}\right)\right\rangle+\left\langle\epsilon^{a}(\mathbf{q}) \boldsymbol{\epsilon}^{b}\left(\mathbf{q}^{\prime}\right)\right\rangle\left\langle\epsilon^{a}(\mathbf{k}-\mathbf{q}) \epsilon^{b}\left(\mathbf{k}^{\prime}-\mathbf{q}^{\prime}\right)\right\rangle\right. \\
& \left.+\left\langle\epsilon^{a}(\mathbf{q}) \epsilon^{b}\left(\mathbf{k}^{\prime}-\mathbf{q}^{\prime}\right)\right\rangle\left\langle\epsilon^{a}(\mathbf{k}-\mathbf{q}) \epsilon^{b}\left(\mathbf{q}^{\prime}\right)\right\rangle\right]|\mathbf{k}-\mathbf{q}|\left|\mathbf{k}^{\prime}-\mathbf{q}^{\prime}\right|(\mathbf{k} \cdot \mathbf{q})\left(\mathbf{k}^{\prime} \cdot \mathbf{q}^{\prime}\right) \int d \tau f(q \tau) f^{\prime}(|\mathbf{k}-\mathbf{q}| \tau) \\
& \times \int d \xi f\left(q^{\prime} \xi\right) f^{\prime}\left(\left|\mathbf{k}^{\prime}-\mathbf{q}^{\prime}\right| \xi\right) .
\end{aligned}
$$

Using Eq. (7), we find

$$
\begin{gathered}
(\mathbf{k} \cdot \mathbf{q})\left(\mathbf{k}^{\prime} \cdot \mathbf{q}^{\prime}\right)\left\langle\epsilon^{a}(\mathbf{q}) \epsilon^{a}(\mathbf{k}-\mathbf{q})\right\rangle\left\langle\epsilon^{b}\left(\mathbf{q}^{\prime}\right) \boldsymbol{\epsilon}^{b}\left(\mathbf{k}^{\prime}-\mathbf{q}^{\prime}\right)\right\rangle=(\mathbf{k} \cdot \mathbf{q})\left(\mathbf{k}^{\prime} \cdot \mathbf{q}^{\prime}\right) \sum_{a, b} \frac{(2 \pi)^{6}}{A^{2} N^{2} q^{3} q^{33}} \delta_{a a} \delta_{b b} \delta_{D}(\mathbf{k}) \delta_{D}\left(\mathbf{k}^{\prime}\right)=0, \\
(\mathbf{k} \cdot \mathbf{q})\left(\mathbf{k}^{\prime} \cdot \mathbf{q}^{\prime}\right)\left\langle\epsilon^{a}(\mathbf{q}) \epsilon^{b}\left(\mathbf{q}^{\prime}\right)\right\rangle\left\langle\epsilon^{a}(\mathbf{k}-\mathbf{q}) \epsilon^{b}\left(\mathbf{k}^{\prime}-\mathbf{q}^{\prime}\right)\right\rangle=\frac{(2 \pi)^{6}}{N A^{2} q^{3}|\mathbf{k}-\mathbf{q}|^{3}}(\mathbf{k} \cdot \mathbf{q})^{2} \delta_{D}\left(\mathbf{q}+\mathbf{q}^{\prime}\right) \delta_{D}\left(\mathbf{k}+\mathbf{k}^{\prime}\right),
\end{gathered}
$$

and

$$
(\mathbf{k} \cdot \mathbf{q})\left(\mathbf{k}^{\prime} \cdot \mathbf{q}^{\prime}\right)\left\langle\epsilon^{a}(\mathbf{q}) \epsilon^{b}\left(\mathbf{k}^{\prime}-\mathbf{q}^{\prime}\right)\right\rangle\left\langle\epsilon^{a}(\mathbf{k}-\mathbf{q}) \epsilon^{b}\left(\mathbf{q}^{\prime}\right)\right\rangle=\frac{(2 \pi)^{6}}{N A^{2} q^{3}|\mathbf{k}-\mathbf{q}|^{3}}(\mathbf{k} \cdot \mathbf{q})(\mathbf{k} \cdot(\mathbf{k}-\mathbf{q})) \delta_{D}\left(\mathbf{q}^{\prime}-\mathbf{q}+\mathbf{k}\right) \delta_{D}\left(\mathbf{k}+\mathbf{k}^{\prime}\right)
$$

Substituting these expressions into Eq. (A1), we obtain

$$
\begin{aligned}
P^{\sigma}(k)= & \frac{C^{2} \eta^{4}}{A^{2} N} \int \frac{d^{3} q}{(2 \pi)^{3}}\left[\left(\int d \tau \frac{f(q \tau) f^{\prime}(|\mathbf{k}-\mathbf{q}| \tau)}{q^{3 / 2}|\mathbf{k}-\mathbf{q}|^{1 / 2}}\right)^{2}(\mathbf{k} \cdot \mathbf{q})^{2}+\left(\int d \tau \frac{f(q \tau) f^{\prime}(|\mathbf{k}-\mathbf{q}| \tau)}{q^{3 / 2}|\mathbf{k}-\mathbf{q}|^{1 / 2}}\right)\left(\int d \xi \frac{f(|\mathbf{k}-\mathbf{q}| \xi) f^{\prime}(q \xi)}{|\mathbf{k}-\mathbf{q}|^{3 / 2} q^{1 / 2}}\right)\right. \\
& \times(\mathbf{k} \cdot \mathbf{q})(\mathbf{k} \cdot(\mathbf{k}-\mathbf{q}))] \\
= & \frac{C^{2} \eta^{4}}{A^{2}} \frac{k}{N} \int \frac{d^{3} v}{(2 \pi)^{3}} I(v,|\hat{\mathbf{k}}-\mathbf{v}|)\left[(\hat{\mathbf{k}} \cdot \mathbf{v})^{2} I(v,|\hat{\mathbf{k}}-\mathbf{v}|)+(\hat{\mathbf{k}} \cdot \mathbf{v})(1-\hat{\mathbf{k}} \cdot \mathbf{v}) I(|\hat{\mathbf{k}}-\mathbf{v}|, v)\right],
\end{aligned}
$$

where $I(a, b)$ is defined in Eq. (18). From here we can then introduce $g_{2}$ in Sec. IV. 


\section{B. The bispectrum}

The bispectrum is obtained by starting with,

$$
\begin{aligned}
\left\langle\delta\left(\mathbf{k}_{1}\right) \delta\left(\mathbf{k}_{2}\right) \delta\left(\mathbf{k}_{3}\right)\right\rangle= & -C^{3} \eta^{6} \int \frac{d^{3} q_{1} d^{3} q_{2} d^{3} q_{3}}{(2 \pi)^{9}}\left\langle\boldsymbol{\epsilon}^{a}\left(\mathbf{q}_{1}\right) \boldsymbol{\epsilon}^{a}\left(\mathbf{k}_{1}-\mathbf{q}_{1}\right) \boldsymbol{\epsilon}^{b}\left(\mathbf{q}_{2}\right) \boldsymbol{\epsilon}^{b}\left(\mathbf{k}_{2}-\mathbf{q}_{2}\right) \boldsymbol{\epsilon}^{c}\left(\mathbf{q}_{3}\right) \boldsymbol{\epsilon}^{c}\left(\mathbf{k}_{3}-\mathbf{q}_{3}\right)\right\rangle \\
& \times\left|\mathbf{k}_{1}-\mathbf{q}_{1}\right|\left|\mathbf{k}_{2}-\mathbf{q}_{2}\right|\left|\mathbf{k}_{3}-\mathbf{q}_{3}\right|\left(\mathbf{k}_{1} \cdot \mathbf{q}_{1}\right)\left(\mathbf{k}_{2} \cdot \mathbf{q}_{2}\right)\left(\mathbf{k}_{3} \cdot \mathbf{q}_{3}\right) \int d \tau_{1} f\left(q_{1} \tau_{1}\right) f^{\prime}\left(\left|\mathbf{k}_{1}-\mathbf{q}_{1}\right| \tau_{1}\right) \\
& \times \int d \tau_{2} f\left(q_{2} \tau_{2}\right) f^{\prime}\left(\left|\mathbf{k}_{2}-\mathbf{q}_{2}\right| \tau_{2}\right) \int d \tau_{3} f\left(q_{3} \tau_{3}\right) f^{\prime}\left(\left|\mathbf{k}_{3}-\mathbf{q}_{3}\right| \tau_{3}\right) .
\end{aligned}
$$

The expectation value of the product of six $\epsilon^{a}(\mathbf{k})$ 's can be expanded with Wick contractions and, after some algebra, and using Eq. (7), we find

$$
\begin{aligned}
\left\langle\delta\left(\mathbf{k}_{1}\right) \delta\left(\mathbf{k}_{2}\right) \delta\left(\mathbf{k}_{3}\right)\right\rangle= & -(2 \pi)^{3} \delta_{D}\left(\mathbf{k}_{1}+\mathbf{k}_{2}+\mathbf{k}_{3}\right) \frac{C^{3} \eta^{6}}{A^{3} N^{2}} \int \frac{d^{3} v}{(2 \pi)^{3}}(\hat{\mathbf{k}} \cdot \mathbf{v}) I(v, b)\left\{\left[(\mathbf{u} \cdot \mathbf{v}) I\left(v, b_{2}\right)-\left(\mathbf{u} \cdot \mathbf{b}_{2}\right) I\left(b_{2}, v\right)\right]\right. \\
& \times\left[((\hat{\mathbf{k}}+\mathbf{u}) \cdot \mathbf{b}) I\left(b, b_{2}\right)+\left((\hat{\mathbf{k}}+\mathbf{u}) \cdot \mathbf{b}_{2}\right) I\left(b_{2}, b\right)\right]+\left[\left(\mathbf{u} \cdot \mathbf{b} I\left(b, b_{12}\right)-\left(\mathbf{u} \cdot \mathbf{b}_{12}\right) I\left(b_{12}, b\right)\right]\right. \\
& \left.\times\left[((\hat{\mathbf{k}}+\mathbf{u}) \cdot \mathbf{v}) I\left(v, b_{12}\right)+\left((\hat{\mathbf{k}}+\mathbf{u}) \cdot \mathbf{b}_{12}\right) I\left(b_{12}, v\right)\right]\right\},
\end{aligned}
$$

where $k \equiv\left|\mathbf{k}_{1}\right|, \hat{\mathbf{k}} \equiv \mathbf{k}_{1} / k, \mathbf{u} \equiv \mathbf{k}_{2} / k$, and we have defined $\mathbf{b} \equiv \hat{\mathbf{k}}-\mathbf{v}, \mathbf{b}_{2} \equiv \mathbf{u}+\mathbf{v}, \mathbf{b}_{12} \equiv \hat{\mathbf{k}}+\mathbf{u}-\mathbf{v}$. Defining $g_{3}$ as in Eq. (25) and performing a change of variables $\mathbf{v} \rightarrow(\hat{\mathbf{k}}-\mathbf{v})$ in the second term of the above integral, we then find

$$
\begin{aligned}
g_{3}\left(k_{1}, k_{2}, k_{3}\right)= & \int \frac{d^{3} v}{(2 \pi)^{3}}[(\hat{\mathbf{k}} \cdot \mathbf{v}) I(v, b)+(\hat{\mathbf{k}} \cdot \mathbf{b}) I(b, v)]\left[(\mathbf{u} \cdot \mathbf{v}) I\left(v, b_{2}\right)-\left(\mathbf{u} \cdot \mathbf{b}_{2}\right) I\left(b_{2}, v\right)\right] \\
& \times\left[\mathbf{b} \cdot(\hat{\mathbf{k}}+\mathbf{u}) I\left(b, b_{2}\right)+\mathbf{b}_{2} \cdot(\hat{\mathbf{k}}+\mathbf{u}) I\left(b_{2}, b\right)\right] .
\end{aligned}
$$

If the upper limit of the integral defining $I(a, b)$ is much greater than one (as expected for subhorizon modes), such that $I(a, b)=I(b, a)$, we then obtain

$$
\begin{aligned}
g_{3}= & \int \frac{d^{3} v}{(2 \pi)^{3}} I(v, b) I\left(v, b_{2}\right) I\left(b, b_{2}\right)(\hat{\mathbf{k}} \cdot(\mathbf{v}-\mathbf{b})) \\
& \times\left(\mathbf{u} \cdot\left(\mathbf{v}+\mathbf{b}_{2}\right)\right)\left((\hat{\mathbf{k}}+\mathbf{u}) \cdot\left(\mathbf{b}-\mathbf{b}_{2}\right)\right) .
\end{aligned}
$$

Finally, simple algebraic rearrangements in Eq. (A9) then results in the far simpler expression, in Eq. (26) for $g_{3}$,

$$
g_{3}=\int \frac{d^{3} v}{(2 \pi)^{3}} H(\mathbf{u}+\mathbf{v}, \mathbf{v}) H(\mathbf{v}, \hat{\mathbf{k}}-\mathbf{v}) H(\hat{\mathbf{k}}-\mathbf{v}, \mathbf{u}+\mathbf{v})
$$

with $H(a, b) \equiv\left(b^{2}-a^{2}\right) I(a, b) \leq 0$. This expression is indeed equivalent to Eq. (59) in Ref. [23], although it is written in a much more compact and simpler way. Note that it is smaller by a factor $(2 \pi)^{3}$ than that of Ref. [23], as a consequence of different conventions.

\section{Some integrals and approximations}

Once modes are well inside the horizon, the upper limit for the integral in Eq. (18) is large, and the integral can then be approximated by

$$
I(a, b) \equiv \int_{0}^{\infty} d s \frac{f(a s) f^{\prime}(b s)}{a^{3 / 2} b^{1 / 2}},
$$

where $f(x)=x^{1 / 2-\alpha} J_{1+\alpha}$, and $\alpha=2$ for matter domination. The integral can be performed analytically; the result is

$$
I(a, b)= \begin{cases}\frac{1}{b^{3}} F\left(a^{2} / b^{2}\right), & \text { for } a<b, \\ -\frac{1}{a^{3}} F\left(b^{2} / a^{2}\right), & \text { for } a>b,\end{cases}
$$

where

$$
\begin{gathered}
F(x) \equiv \frac{3 \sqrt{\pi}}{4^{n}}\left[\frac{x_{2} F_{1}\left(\frac{5}{2}, \frac{5}{2}-n ; n+2 ; x\right)}{\Gamma(n+2) \Gamma\left(n-\frac{3}{2}\right)}\right. \\
\left.-\frac{2 F_{1}\left(\frac{5}{2}, \frac{3}{2}-n ; n+1 ; x\right)}{\Gamma(n+1) \Gamma\left(n-\frac{1}{2}\right)}\right],
\end{gathered}
$$

${ }_{2} F_{1}(w, x ; y ; z)$ is the hypergeometric function, and $n=$ $1+\alpha$ is the order of the Bessel function. While straightforward to evaluate numerically, this exact solution may be computationally expensive to evaluate repeatedly. We therefore use for our numerical work the approximation,

$$
I(a, b) \simeq \begin{cases}\frac{-1}{96 b^{3}} \frac{(b / a)^{\kappa}-1}{(b / a)^{\kappa}+1}, & \text { if } a<b, \\ \frac{1}{96 a^{3}} \frac{(a / b)^{\kappa}-1}{(a / b)^{\kappa}+1}, & \text { if } b<a,\end{cases}
$$

which provides good agreement with the exact results with $\kappa=2.5$. 
[1] A. H. Guth and S. Y. Pi, Phys. Rev. Lett. 49, 1110 (1982); A. A. Starobinsky, Phys. Lett. 117B, 175 (1982); J. M. Bardeen, P. J. Steinhardt, and M. S. Turner, Phys. Rev. D 28, 679 (1983).

[2] T. J. Allen, B. Grinstein, and M. B. Wise, Phys. Lett. B 197, 66 (1987); L. A. Kofman and D. Y. Pogosian, Phys. Lett. B 214, 508 (1988); D. S. Salopek, J. R. Bond, and J. M. Bardeen, Phys. Rev. D 40, 1753 (1989); A. D. Linde and V. F. Mukhanov, Phys. Rev. D 56, R535 (1997); P. J. E. Peebles, Astrophys. J. 510, 523 (1999); 510, 531 (1999).

[3] S. Mollerach, Phys. Rev. D 42, 313 (1990); D. H. Lyth and D. Wands, Phys. Lett. B 524, 5 (2002); T. Moroi and T. Takahashi, Phys. Lett. B 522, 215 (2001); 539, 303(E) (2002); D. H. Lyth, C. Ungarelli, and D. Wands, Phys. Rev. D 67, 023503 (2003); K. Ichikawa et al., Phys. Rev. D 78, 023513 (2008); K. Enqvist, S. Nurmi, O. Taanila, and T. Takahashi, J. Cosmol. Astropart. Phys. 04 (2010) 009; K. Enqvist and T. Takahashi, J. Cosmol. Astropart. Phys.12 (2009) 001; 09 (2008) 012; K. Enqvist and S. Nurmi, J. Cosmol. Astropart. Phys. 10 (2005) 013; A. L. Erickcek, M. Kamionkowski, and S. M. Carroll, Phys. Rev. D 78, 123520 (2008); A. L. Erickcek, C. M. Hirata, and M. Kamionkowski, Phys. Rev. D 80, 083507 (2009).

[4] G. R. Dvali and S. H. H. Tye, Phys. Lett. B 450, 72 (1999); P. Creminelli, J. Cosmol. Astropart. Phys. 10 (2003) 003; M. Alishahiha, E. Silverstein, and D. Tong, Phys. Rev. D 70, 123505 (2004).

[5] A. Vilenkin and E.P.S. Shellard, Cosmic Strings and Other Topological Defects (Cambridge University Press, Cambridge, England, 1994); R. Durrer, New Astron. Rev. 43, 111 (1999); R. Durrer, M. Kunz, and A. Melchiorri, Phys. Rep. 364, 1 (2002).

[6] S. Sarangi and S. H. H. Tye, Phys. Lett. B 536, 185 (2002); N. T. Jones, H. Stoica, and S. H. H. Tye, Phys. Lett. B 563, 6 (2003); E. J. Copeland, R. C. Myers, and J. Polchinski, J. High Energy Phys. 06 (2004) 013; G. Dvali and A. Vilenkin, J. Cosmol. Astropart. Phys. 03 (2004) 010; R. Jeannerot, J. Rocher, and M. Sakellariadou, Phys. Rev. D 68, 103514 (2003); L. Kofman, A. D. Linde, and A. A. Starobinsky, Phys. Rev. Lett. 76, 1011 (1996); G. N. Felder, J. Garcia-Bellido, P. B. Greene, L. Kofman, A. D. Linde, and I. Tkachev, Phys. Rev. Lett. 87, 011601 (2001); G. N. Felder, L. Kofman, and A. D. Linde, Phys. Rev. D 64, 123517 (2001).

[7] N. Turok and D. N. Spergel, Phys. Rev. Lett. 66, 3093 (1991); M. Kunz and R. Durrer, Phys. Rev. D 55, R4516 (1997).

[8] N. Bevis, M. Hindmarsh, and M. Kunz, Phys. Rev. D 70, 043508 (2004).

[9] P. Crotty, J. Garcia-Bellido, J. Lesgourgues, and A. Riazuelo, Phys. Rev. Lett. 91, 171301 (2003); M. Beltran, J. Garcia-Bellido, J. Lesgourgues, and M. Viel, Phys. Rev. D 72, 103515 (2005).

[10] M. Cruz, N. Turok, P. Vielva, E. Martinez-Gonzalez, and M. Hobson, Science 318, 1612 (2007); C. L. Bennett et al., arXiv:1001.4758.

[11] N. Bartolo et al., Phys. Rep. 402, 103 (2004).

[12] F. Bernardeau, S. Colombi, E. Gaztanaga, and R. Scoccimarro, Phys. Rep. 367, 1 (2002).
[13] T. Falk, R. Rangarajan, and M. Srednicki, Astrophys. J. 403, L1 (1993); A. Gangui et al., Astrophys. J. 430, 447 (1994); A. Gangui, Phys. Rev. D 50, 3684 (1994).

[14] L. M. Wang and M. Kamionkowski, Phys. Rev. D 61, 063504 (2000).

[15] J. M. Maldacena, J. High Energy Phys. 05 (2003) 013; V. Acquaviva, N. Bartolo, S. Matarrese, and A. Riotto, Nucl. Phys. B667, 119 (2003).

[16] D. Babich, P. Creminelli, and M. Zaldarriaga, J. Cosmol. Astropart. Phys. 08 (2004) 009; P. Creminelli et al., J. Cosmol. Astropart. Phys. 05 (2006) 004; 03 (2007) 005.

[17] S. Hannestad, T. Haugboelle, P. R. Jarnhus, and M. S. Sloth, arXiv:0912.3527.

[18] X. c. Luo, Astrophys. J. 427, L71 (1994); L. Verde et al., Mon. Not. R. Astron. Soc. 313, L141 (2000); E. Komatsu and D. N. Spergel, Phys. Rev. D 63, 063002 (2001).

[19] P. Coles et al., Mon. Not. R. Astron. Soc. 264, 749 (1993); X.c. Luo and D. N. Schramm, Astrophys. J. 408, 33 (1993); E. Lokas et al., Mon. Not. R. Astron. Soc. 274, 730 (1995); M. J. Chodorowski and F. R. Bouchet, Mon. Not. R. Astron. Soc. 279, 557 (1996); A. J. Stirling and J. A. Peacock, Mon. Not. R. Astron. Soc. 283, L99 (1996); R. Durrer et al., Phys. Rev. D 62, 021301 (2000); L. Verde and A.F. Heavens, Astrophys. J. 553, 14 (2001); A. Buchalter and M. Kamionkowski, Astrophys. J. 521, 1 (1999); A. Buchalter, M. Kamionkowski, and A. H. Jaffe, Astrophys. J. 530, 36 (2000).

[20] W. A. Chiu, J. P. Ostriker, and M. A. Strauss, Astrophys. J. 494, 479 (1998); J. Robinson, E. Gawiser, and J. Silk, Astrophys. J. 532, 1 (2000); J. A. Willick, Astrophys. J. 530, 80 (2000); L. Verde et al., Mon. Not. R. Astron. Soc. 321, L7 (2001); N. N. Weinberg and M. Kamionkowski, Mon. Not. R. Astron. Soc. 341, 251 (2003); S. Matarrese, L. Verde, and R. Jimenez, Astrophys. J. 541, 10 (2000); L. Verde et al., Mon. Not. R. Astron. Soc. 325, 412 (2001); M. LoVerde et al., J. Cosmol. Astropart. Phys. 04 (2008) 014.

[21] M. Kamionkowski, L. Verde, and R. Jimenez, J. Cosmol. Astropart. Phys. 01 (2009) 010.

[22] N. Dalal et al., Phys. Rev. D 77, 123514 (2008); S. Matarrese and L. Verde, Astrophys. J. 677, L77 (2008); A. Slosar et al., J. Cosmol. Astropart. Phys. 08 (2008) 031; C. Carbone, L. Verde, and S. Matarrese, Astrophys. J. 684, L1 (2008).

[23] A. H. Jaffe, Phys. Rev. D 49, 3893 (1994).

[24] A. Silvestri and M. Trodden, Phys. Rev. Lett. 103, 251301 (2009); M. Hindmarsh, C. Ringeval, and T. Suyama, Phys. Rev. D 80, 083501 (2009); 81, 063505 (2010); D. M. Regan and E.P.S. Shellard, arXiv:0911.2491.

[25] M. Kamionkowski and J. March-Russell, Phys. Lett. B 282, 137 (1992); M. Kamionkowski and J. March-Russell, Phys. Rev. Lett. 69, 1485 (1992); R. Holman, S. D. H. Hsu, T. W. Kephart, E. W. Kolb, R. Watkins, and L. M. Widrow, Phys. Lett. B 282, 132 (1992); R. Holman, S. D. H. Hsu, E. W. Kolb, R. Watkins, and L. M. Widrow, Phys. Rev. Lett. 69, 1489 (1992).

[26] S. M. Barr and D. Seckel, Phys. Rev. D 46, 539 (1992).

[27] S. Veeraraghavan and A. Stebbins, Astrophys. J. 365, 37 (1990).

[28] U. L. Pen, U. Seljak, and N. Turok, Phys. Rev. Lett. 79, 1611 (1997). 
[29] U.L. Pen, D. N. Spergel, and N. Turok, Phys. Rev. D 49, 692 (1994).

[30] P. Creminelli, A. Nicolis, L. Senatore, M. Tegmark, and M. Zaldarriaga, J. Cosmol. Astropart. Phys. 05 (2006) 004.

[31] E. Komatsu et al. (WMAP Collaboration), Astrophys. J. Suppl. Ser. 148, 119 (2003); A.P. S. Yadav and B.D. Wandelt, Phys. Rev. Lett. 100, 181301 (2008); E. Komatsu et al. (WMAP Collaboration), Astrophys. J. Suppl. Ser. 180, 330 (2009).

[32] N. Turok, U.L. Pen, and U. Seljak, Phys. Rev. D 58, 023506 (1998).

[33] E. Komatsu et al., arXiv:1001.4538.

[34] A. Cooray, D. Sarkar, and P. Serra, Phys. Rev. D 77, 123006 (2008).

[35] R. Durrer, M. Kunz, and A. Melchiorri, Phys. Rev. D 59, 123005 (1999).
[36] U. Seljak, U. L. Pen, and N. Turok, Phys. Rev. Lett. 79, 1615 (1997).

[37] M. Kamionkowski, A. Kosowsky, and A. Stebbins, Phys. Rev. D 55, 7368 (1997); Phys. Rev. Lett. 78, 2058 (1997); M. Zaldarriaga and U. Seljak, Phys. Rev. D 55, 1830 (1997); U. Seljak and M. Zaldarriaga, Phys. Rev. Lett. 78, 2054 (1997).

[38] D. Baumann and M. Zaldarriaga, J. Cosmol. Astropart. Phys. 06 (2009) 013; P. Adshead and E. A. Lim, arXiv:0912.1615.

[39] L. M. Krauss, Phys. Lett. B 284, 229 (1992); E. Fenu, D. G. Figueroa, R. Durrer, and J. Garcia-Bellido, J. Cosmol. Astropart. Phys. 10 (2009) 005; K. JonesSmith, L. M. Krauss, and H. Mathur, Phys. Rev. Lett. 100, 131302 (2008). 